\title{
Confirmed child patient with covid-19 infection, opperated for associated surgical pathology - first pediatric case in Romania
}

\author{
DANIEL-OVIDIU COSTEA ${ }^{1}$, FLORIN-DANIEL ENACHE ${ }^{1}$, RADU BAZ ${ }^{1}$, ADRIAN PAUL \\ SUCEVEANU ${ }^{1}$, ANDRA I. SUCEVEANU ${ }^{1}$, VALERIU ARDELEANU ${ }^{1,2,3}$, LAURA MAZILU ${ }^{1}$, \\ ANDREEA CRISTINA COSTEA ${ }^{5}$, FLORIN BOTEA $^{4}$, FELIX VOINEA $^{1}$ \\ 1'The Faculty of Medicine "OVIDIUS”, Campus, Aleea Universității, nr. 1, Corp B, Constanța, \\ Constanța County, Romania \\ ${ }^{2}$ Dunărea de Jos” University, Str. Domnească, nr. 47, Galați, 800008, Galați County, Romania \\ ${ }^{3}$ General Hospital CFR, Str. Alexandru Moruzzi, nr. 5-7, Galați, 800223, Romania \\ ${ }^{4}$ Fundeni Clinical Institute, Șos. Fundeni, nr. 258, Sector 2, Bucharest, Romania \\ ${ }_{5}^{5}$ iaverum Constanța Dialysis and Nephrology Center, Str. Nicolae Iorga, nr. 20, Constanța, Romania
}

\begin{abstract}
Background: The year 2020 will remain in history as a challenge for the humanity due to the pandemic situation caused by the Coronavirus - COVID-19 disease. The virus spread rapidly throughout the world, affecting people of all ages including children.

Objectives: The purpose of the research was to present the first case in Romania of a child infected with COVID-19, operated for associated surgical pathology.

Materials and Methods: The case presented in this paper is a 6 years and 9 months old female child with COVID-19 infection admitted in the Clinic of Pediatric Surgery of Constanta Emergency County Hospital and operated for a peritonitis with appendicular abscess. In the last 7 days she presented transient abdominal pain with episodes of fever that referred to antipyretics.

Results: From a surgical point of view, the case is ordinary, without possible redoubtable complications but the clinical picture was atypical due to coronavirus infection. The viral infection did not have a symptomatic history, as in most adult cases, but the lung x-ray showed infectious changes, which is why she was tested for potential COVID-19 infection, with a positive result.

Conclusion: The case showed the clinical picture of atypical coronavirus infection in children. Viral infections in children have more abdominal tropism, with mesenteric lymphadenitis, which in some cases can lead to appendicular inflammation with secondary appendicitis. Undiagnosed in time, it can be complicated by peritonitis and any other type of appendicular infectious pathology.
\end{abstract}

Keywords Coronavirus disease, COVID-19 infection, child, peritonitis, appendicular abscess.

To cite this article: COSTEA DO, ENACHE FD, BAZ R, SUCEVEANU AP, SUCEVEANU AI, ARDELEANU V, MAZILU L, COSTEA AC, BOTEA F, VOINEA F. Confirmed child patient with covid-19 infection, opperated for associated surgical pathology - first pediatric case in Romania. Rom Biotechnol Lett. 2020; 25(6): 2107-2110. DOI: 10.25083/rb1/25.6/2107.2110 


\section{Introduction}

The recent coronavirus disease (COVID-19), caused by severe acute respiratory syndrome coronavirus 2 (SARS-CoV-2), spreads rapidly around the globe [1]. It originated in Wuhan, in the Hubei Province of China. There are about 5 million confirmed cases around the world since the outbreak in 2019. The Chinese Center for Disease Control and Prevention say that infected children aged 19 and younger are only $2.16 \%$ of all confirmed cases [2].

Because of high number of coronavirus cases and because of the medical resources a lot of children with mild infections or asymptomatic might not have been tested [3]. This might alter the real number of infected children with COVID-19 and also will affect the diagnosis and management of children admitted for surgical diseases.

This was the real situation in Romania, when at the beginning of COVID-19 pandemics, because of delayed supply of materials and relative shortage in medical resources, children infected with the new coronavirus were diagnosed with difficulty, especially those with surgical problems, with associated digestive symptoms. As an example we will present a similar case, the first one in Romania, a 6 years and 9 months old girl, admitted in March 2020, in the Clinic of Pediatric Surgery and Orthopedics of Constanta Emergency County Hospital and treated for acute surgical abdomen-peritonitis due to an appendicular abscess, previously confirmed with COVID-19 infection.

\section{Materials and Methods}

At the end of March 2020, a 6 years and 9 months old girl came with her mother in the Emergency Room of Constanta County Hospital accusing abdominal pain,

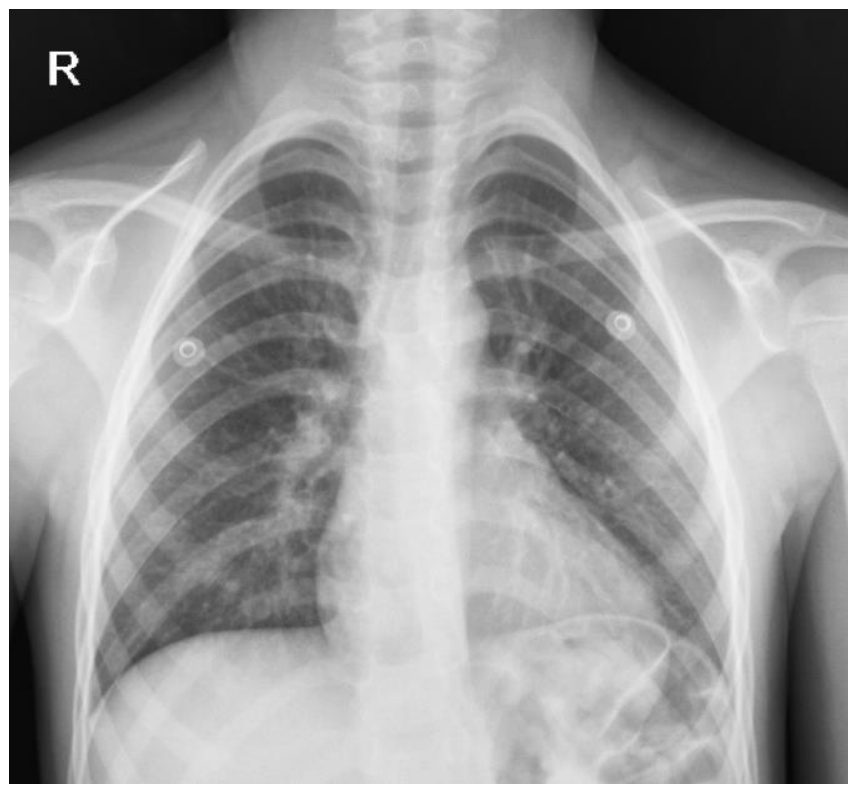

Figure 1. Pulmonary Xray showing bilateral pulmonary microopacity. associated with nausea, vomiting and diarrhea. Body temperature under the armpit was $37.8^{\circ} \mathrm{C}$.

A week ago, same child came in the Emergency Room, accusing same, but mild abdominal pain, with no sign of acute surgical abdomen. She was discharged with diagnosis of an abdominal viral infection and with symptomatic treatment at home.

At her second arrival in the Emergency Room she was examined respecting all the rules of protection of the medical staff and implicitly of the patient. She was very scared and noncompliant with voluntary contraction of abdominal muscles. During the anamnesis we found that she had recently contact with persons returned from areas at high risk of COVID-19 infection - mother had returned from Germany 3 weeks ago and the father a week ago (they had been quarantined 14 days).

Due to her history and the altered radiological image of the lungs (Figure 1), we decided to test her for possible COVID-19 infection. The rapid test came out positive. Following the national protocol, the competent authorities and the management of the hospital were announced, who were very prompt, providing us with all the necessary protection materials. Due to the associated abdominal symptoms, we had to hospitalize the child for further investigations and specialized treatment in the Clinic of Pediatric Surgery, in an isolated room, specially designed for patients with COVID-19 infection.

At admission, blood tests showed leukocytosis with neutrophilia, monocytosis and lymphopenia; increased inflammatory samples (RCP, fibrinogen, ESR). Abdominal ultrasound did not show the appendix and the appendicular area, due to intestinal flatulence, but only a blade of fluid in the Douglas space.

Due to the symptoms of possible acute surgical abdomen and fever, we instituted broad-spectrum antibiotic and symptomatic treatment.

Abdominal CT scan was performed and showed a vermiform appendix in retrocecal position with circumferentially and uniform thickened wall, intraluminal, with a formation with limestone density with maximum diameters of $6 / 4 / 8 \mathrm{~mm}$ that obstructs the lumen at the base, with an apparent solution of continuity in the middle third, where it comes contiguous with a collection of parafluid density and aerial inclusions, which has maximum diameters of 28/23/34 $\mathrm{mm}$ situated between the parietal peritoneum and the posterior face of the cecum, which associates the uniform thickening of the adjacent parietal peritoneum; multiple lymphadenopathies with maximum axial diameters of $17 / 10 \mathrm{~mm}$ in the mesentery and especially in the ileocolic space (Figure 2, A\&B). 

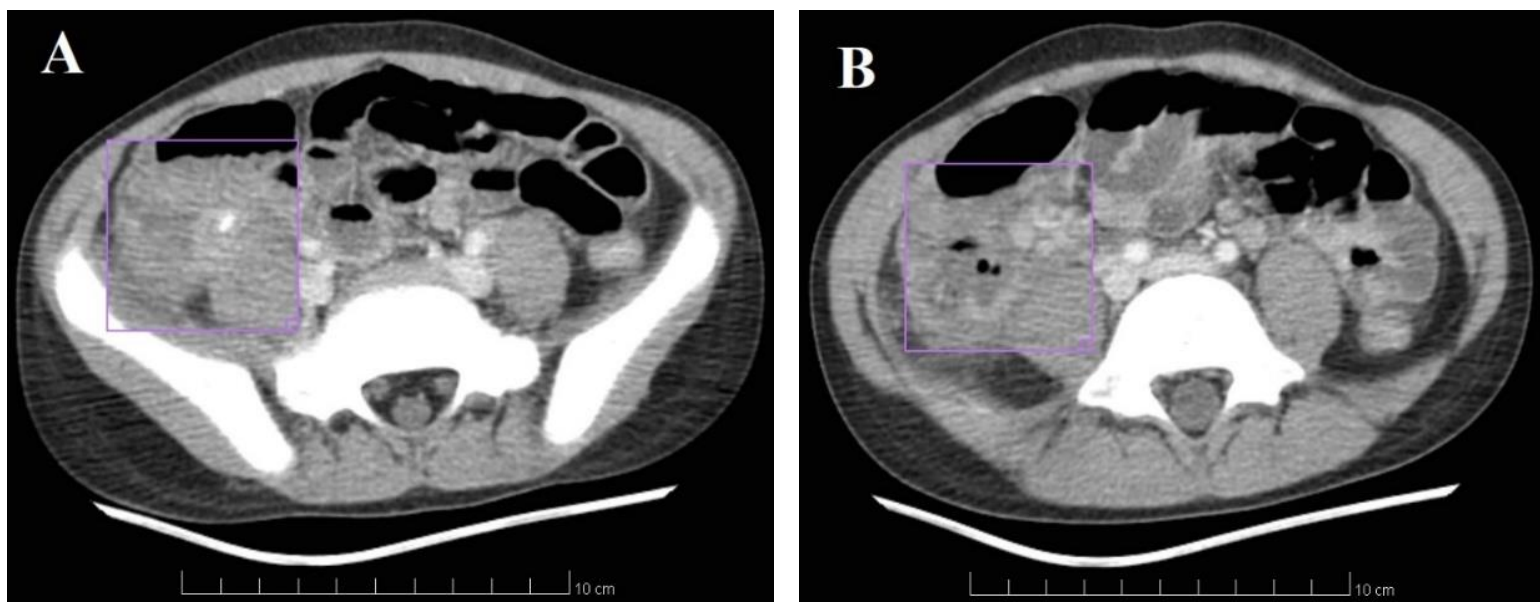

Figure 2. A\&B. Abdominal CT scan showing (A) the appendix with a calcified coprolite inside and (B) a collection of parafluid density and aerial inclusions with multiple adjacent lymphadenopathies.

The RT-PCR test for COVID-19 infection confirms the diagnosis of coronavirus infection. Following imaging investigations and the symptoms of acute surgical abdomen, surgical intervention is decided.

Surgery was performed by a team headed by pediatric surgeon Florin-Daniel Enache, MD, PhD, taking all measures to protect against COVID-19 infection. The postoperative diagnosis was acute perforated gangrenous appendicitis, with coprolite, retrocaecal, with periapendicular abscess. We took a sample of pus for culture and DST (antibiogram). Two types of germs have developed Citrobacter freundii and Enterococcus avium. The antibiotic therapy continued postoperatory. Also, the antiviral therapy was instituted - Kaletra (Lopinavir $80 \mathrm{mg}$ and Ritonavir $20 \mathrm{mg} / \mathrm{ml}$ ). The peritoneal cavity was drained for 72 hours. When the drainage tube was removed, a sample from its tip was taken for culture. The result was negative, no germs on the medium culture.

Postoperative evolution was favorable, with antibiotic, antiviral and symptomatic treatment. The sutures were removed 7 days postoperatively. The patient was tested again twice, with RT-PCR test for COVID-19 infection, both of them being negative. One week after the operation, she was discharged with the obligation to remain isolated at home with her mother for 14 days. We mention that the mother was tested, being negative for COVID-19 infection.

\section{Results and Discussions}

About 4.8 million confirmed cases and about 0.3 million deaths from new coronavirus infection have been reported in the world since the outbreak in Wuhan, China, in December, 2019 [4, 5]. Until present day, most of the cases and mortality have been seen among adult population, especially the elderly.

Sometimes children are asymptomatic carriers and usually have milder clinical manifestations [6]. At the beginning of pandemic outbreak, gastrointestinal symptoms have not been recognized and rarely reported in the literature [7]. In patients with acute respiratory syndrome, with a high level of viral load in the nasopharynx, diarrhea was a frequent symptom [8]. In the United Kingdom have been reported aspects of an inflammatory syndrome related to COVID infection in children with gastrointestinal symptoms $[9,10]$.

There are other studies that show gastrointestinal implications related with appendicitis in children infected with SARS-CoV-2 [11, 12]. The case above is eloquent in this context.

The girl treated in our Clinic was admitted for gastrointestinal symptomatology and positive for COVID19 infection. We instituted a nonoperative management for uncomplicated appendicitis, solution adopted in the literature in case of associated infection with COVID-19 [13]. This will avoid aerosolization during general anesthesia and also avoids the anesthesia process in children with respiratory symptoms.

After several other investigations, especially abdominal CT scan, we decided to operate the child, as we were not dealing with an uncomplicated appendicitis, but one with an abscess. When we performed surgery personal protective equipment was used and minimum necessary staff participated, as recommended in the literature $[14,15]$. We adopted the classic technique, not laparoscopy, because laparoscopy carries a theoretical risk of viral transmission through aerosolization [16].

\section{Conclusions}

In children, COVID-19 infection has abdominal symptoms similar to other viral infections associated with mesenteric lymphadenitis. Viral infections with abdominal tropism should be kept under observation because they can add bacterial infections, especially due to the presence of periapendicular lymphadenopathy that can 
block the lumen of the appendix by extrinsic compression and resulting in appendicitis evolving to gangrene, perforation and appendicular peritonitis, with or without periapendicular abscess.

So in this pandemic period in the Emergency Room we recommend to increase the awareness of this clinical presentation, particularly of those with associated signs on the pulmonary Xray.

For this type of clinical presentation we strongly recommend minimum blood tests (blood cells count and inflammatory tests), abdominal ultrasound, chest Xray and for sure a swab for SARS-CoV-2 PCR, before admission and any other treatment decisions.

\section{References}

1. M. MARTINEZ-ALVAREZ, A. JARDE, E. USUF et al. COVID-19 pandemic in West Africa. Lancet Glob Health 2020; 8:e631-2.

2. W. ZUNYOU, J.M. McGOOGAN. Characteristics of and important lessons from the coronavirus disease 2019 (COVID-19) outbreak in China: summary of a report of 72314 cases from the Chinese center for disease control and prevention. JAMA (2020).

3. A. CRUZ, S. ZEICHNER. COVID-19 in children: initial characterization of the pediatric disease. Pediatrics (2020).

4. Johns Hopkins University and Medicine. COVID-19 dashboard by the Center for Systems Science and Engineering at Johns Hopkins University. https://coronavirus.jhu.edu/map.html (accessed on May 18, 2020).

5. A.CERIEllo, A. PANTEA STOIAN, M. RIZZO. COVID-19 and diabetes management: what should be considered?, Diabetes Research and Clinical Practice (2020), doi: https://doi.org/10.1016/j.diabres.2020. 108151 [Epub ahead of print].

6. K. SHEN, Y. YANG, T. WANG et al. Diagnosis, treatment and prevention of 2019 novel coronavirus infection in children: experts' consensus statement. World J Pediatr (2020), published online Feb 7.

7. L. LI, W. WU, S. CHEN et al. Digestive system involvement of novel coronavirus infection: prevention and control infection from a gastroenterology perspective. J Dig Dis, 21:199-204 (2020).
8. V.C.C. CHENG, I.F.N. HUNG, B.S.F. TANG et al. Viral replication in the nasopharynx is associated with diarrhea in patients with severe acute respiratory syndrome. Clin Infect Dis, 38: 467-75 (2004).

9. D. CAMPBELL, I. SAMPLE. At least $12 \mathrm{UK}$ children have needed intensive care due to illness linked to COVID-19. April 27, 2020 https://www.theguardian.com/world/2020/apr/27/nhswarns-of-rise-in-children-withnew-illness-that-maybe-linked-tocoronavirus?CMP=Share iOSApp_Other (accessed on April 29, 2020).

10. S. RIPHAGEN, X. GOMEZ, C. GONZALEZMARTINEZ, N. WILKINSON, P. THEOCHARIS. Hyperinflammatory shock in children during COVID-19 pandemic. Lancet 2020; published online May 6.

11. Z. ZHOU, N. ZHAO, Y. SHU, S. HAN, B. CHEN, $X$. SHU. Effect of gastrointestinal symptoms on patients infected with COVID-19. Gastroenterology (2020).

12. D. SUN, H. LI, X-X. LU et al. Clinical features of severe pediatric patients with coronavirus disease 2019 in Wuhan: A single center's observational study. World J Pediatr, 1-9 (2020).

13. L. HUANG, Y. YIN, L. YANG, C. WANG, Y. LI, Z. ZHOU. Comparison of antibiotic therapy and appendectomy for acute uncomplicated appendicitis in children a meta-analysis. JAMA Pediatr, 171:426-434 (2017).

14. American College of Surgeons. Frequently Asked Questions. How do I manage surgery for COVID-19 PUI/confirmed patients? https://www.facs.org/covid19/newsletter/032020/faq (accessed March 24, 2020).

15. M.C.T. DIMITRIU, A. PANTEA-STOIAN, A.C. SMARANDA, A.A. NICA, A.C. CARAP, V.D. CONSTANTIN, A. DAVITOIU, C. CIRSTOVEANU, N. BACALBASA, O.G. BRATU, F. JACOTAALEXE, C.D. BADIU, C.G. SMARANDACHE, B. SOCEA. Burnout syndrome in Romanian medical residents in time of the COVID-19 pandemic, Medical Hypotheses, Volume 144, November 2020, 109972, https://doi.org/10.1016/j.mehy.2020.109972 [Epub ahead of print].

16. M.H. ZHENG, L. BONI, A. FINGERHUT. Minimally invasive surgery and the novel coronavirus outbreak: Lessons learned in China and Italy. Ann Surg (2020) [Epub ahead of print]. 